\title{
Sinonasal inverted papilloma recurrence rates and evaluation of current staging systems*
}

\author{
William Mak, Devon Webb, Salahuddin Al-Salihi, Anali Dadgostar, \\ Amin Javer
}

Division of Otolaryngology, University of British Columbia, St. Paul's Sinus Centre, Vancouver, British Columbia, Canada
Rhinology 56: 4, 407-414, 2018

https://doi.org/10.4193/Rhin18.039

*Received for publication:

February 27, 2018

Accepted: May 26, 2018

Background: Sinonasal inverted papillomas (SNIPs) are benign epithelial growths with high recurrence rates after surgical management. This study aims to evaluate SNIP recurrence rates after endoscopic surgery and to provide a comparison of published staging systems.

Methods: This chart review evaluated primary and revision SNIP cases from January 2008 to December 2016 at a tertiary sinus centre. Data was collected on patient demographics, origin site, surgical approaches, follow-up duration, recurrence, and smoking history. Each case was staged using Krouse, Oikawa, Cannady, Han, and Kamel systems.

Results: 52 primary and 22 revision SNIP patients had a mean follow-up of 42.3 (range:3-55) months. 11 primary cases (21.1\%) and 5 revision cases (22.7\%) had recurrences. Primary and revision cases had a mean time to recurrence of 24.0 (range:3-55) and 14.6 (range:10-20) months respectively. Smoking history had an OR of 0.63 (Cl 95\%: 0.18-2.22) for recurrence. The age group of 20-39 years featured the highest rates of recurrence. Patient groups defined by each staging system were compared by Kaplan-Meier survival analyses and logrank tests. Chi-squared values for Krouse, Oikawa, Cannady, Han, and Kamel systems were 6.73 ( $p>0.05$ ), $7.02(p>0.05), 6.19(p=0.045), 8.23(p=0.042)$ and $3.29(p>0.05)$ respectively.

Conclusion: Recurrence rates found in this study are comparable to published literature. No statistical significance was found to associate smoking with recurrence. Han and Cannady staging systems were found to define patient groups that correlated well with recurrence. Staging systems should play a role in the management of SNIPs, especially to identify patients requiring additional post-surgical monitoring.

Key words: endoscopy, Krouse, staging, sinonasal inverted papilloma, smoking

\section{Introduction}

Sinonasal inverted papillomas (SNIPs) are benign epithelial growths that occur in the nasal cavity or paranasal sinuses ${ }^{(1,2)}$. Patients typically present with nonspecific symptoms including nasal obstruction, rhinorrhea, facial pain/pressure, headache, and hyposmia/anosmia ${ }^{(3,4)}$. The diagnosis is entertained in cases of an unilateral growth in the nasal cavity, which on endoscopy has a characteristic grayish polypoid mass with a multinodular surface ${ }^{(5-7)}$. Histological confirmation of an inverted squamous epithelial growth into the underlying connective tissue stroma, in the presence of an intact basement membrane, is required for diagnosis ${ }^{(6)}$.

The etiology of the growth is unknown ${ }^{(8)}$. Epidemiologic and meta-analysis studies suggest Human Papilloma Virus (HPV), especially HPV-18, and Epstein-Barr Virus as potential causes $(2,9,10)$. Smoking and allergies are other factors which have been suspected. However, a specific cause has yet to be confirmed $(2,11,12)$.

Endoscopic surgical approaches have now become the gold standard for excision of these tumours ${ }^{(2-4)}$. As opposed to the previous open approaches, the endoscopic approach is a less invasive method to evaluate for the presence of carcinoma and remove the mass ${ }^{(2,13)}$. Its tendency for minimal invasiveness is critical given the proximity to the brain, optic nerve, orbit, and vasculature around the surgical site. Damage to these important adjacent structures can result in significant complications ${ }^{(2,14)}$. 
SNIPs are concerning due to their characteristic aggressive local growth and high rates of recurrence after treatment, as well as their ability to convert to more aggressive pathology. 5 to $15 \%$ of SNIPs can progress to squamous cell carcinoma ${ }^{(1,2,15)}$. SNIP staging systems are an important consideration when evaluating a patient's risk of recurrence. The ideal staging system would be one that is able to predict a recurrence well. Currently, the system devised by Krouse et al. is used more than any other ${ }^{(16,17)}$. A meta-analysis by Lisan et al. found that Krouse Stage T3 SNIPs have a significant risk of recurrence compared to $\mathrm{T} 2{ }^{(18)}$. Furthermore, different classification systems and modifications to the Krouse system have been proposed over the years $(1,16,17,19-21)$. A comparison between the Krouse, Cannady, and Han staging systems has been published previously in 2010 (17). This study expands on this idea by including Oikawa and Kamel. The Oikawa staging system subdivides Krouse Stage T3; this may highlight a correlation between difficult to access locations with recurrence risk. It also addresses the varying recurrence rates and heterogeneity of Stage T3 found in the meta-analysis by Lisan et al. ${ }^{(18)}$. Additionally, the inclusion of the Kamel system, which is relatively simple, may provide a rapid assessment tool for recurrence risk. Of all the above systems, only the Cannady system offered "prognostic information in the endoscopic era" (19). Overall, this study aims to provide an updated comparison of the many currently published staging systems, particularly for cases that were primarily treated using endoscopic approaches at a tertiary level referral centre ${ }^{(17)}$.

The purpose of this study is to evaluate SNIP recurrence rates after endoscopic surgery, as well as to provide a comparison of published staging systems by Krouse et al., Oikawa et al., Cannady et al., Han et al., and Kamel et al. (Table 1).

\section{Materials and methods}

The study protocol was approved by the Providence Health Care Research Ethics Board (Ethics number H16-03218). A retrospective chart review of patients with the diagnosis of SNIP between 2008 and 2016 was conducted for SNIP patients treated at St. Paul's Sinus Centre, a tertiary rhinology referral centre. Collected data included patient demographics (including age and gender), smoking history, co-morbidities, follow-up appointments, and recurrences. All cases were histopathologically confirmed SNIPs; carcinomas were excluded. The degree of dysplasia was not available for all cases, and thus not recorded. Patients were evaluated endoscopically and CT imaging of the sinuses was performed in all cases. For the purposes of the study, staging of each SNIP was repeated by a single rhinologist (SA) using archived CT scans found in patient records. He was blinded from the purposes of the study while staging. Furthermore, he evaluated CT scans without patient identifiers to minimize bias. Only CT imaging was available for every case. Using this modality, the cases were staged with each of the six systems.
Table 1. Sinonasal inverted papilloma staging systems.

\begin{tabular}{|c|c|}
\hline \multicolumn{2}{|c|}{ Krouse ${ }^{(16)}$} \\
\hline T1 & SNIP is confined to the nasal cavity \\
\hline $\mathrm{T} 2$ & $\begin{array}{l}\text { SNIP involvement of the ostiomeatal complex, ethmoid } \\
\text { sinus, and/or medial maxillary sinus; with or without invol- } \\
\text { vement of the nasal cavity }\end{array}$ \\
\hline T3 & $\begin{array}{l}\text { SNIP involvement of lateral, inferior, superior, anterior, or } \\
\text { posterior walls of the maxillary sinus, the sphenoid sinus, } \\
\text { and/or the frontal sinus }\end{array}$ \\
\hline $\mathrm{T} 4$ & $\begin{array}{l}\text { SNIP involvement beyond the sinonasal cavities or associ- } \\
\text { ated with malignancy }\end{array}$ \\
\hline \multicolumn{2}{|c|}{ Oikawa (Modified Krouse) ${ }^{(20)}$} \\
\hline T3A & $\begin{array}{l}\text { Krouse T3 SNIP without frontal sinus or supraorbital recess } \\
\text { involvement }\end{array}$ \\
\hline T3B & $\begin{array}{l}\text { Krouse T3 SNIP with frontal sinus or supraorbital recess } \\
\text { involvement }\end{array}$ \\
\hline \multicolumn{2}{|c|}{ Cannady ${ }^{(19)}$} \\
\hline Group A & $\begin{array}{l}\text { SNIP is confined to the nasal cavity, ethmoid sinuses, and/ } \\
\text { or medial maxillary wall }\end{array}$ \\
\hline Group B & $\begin{array}{l}\text { SNIP involvement of any maxillary wall (besides the me- } \\
\text { dial wall), frontal sinus, or sphenoid sinus }\end{array}$ \\
\hline Group C & SNIP involvement beyond the sinonasal cavities \\
\hline \multicolumn{2}{|l|}{ Han (1) } \\
\hline Group I & $\begin{array}{l}\text { SNIP confined to the nasal cavity, lateral nasal wall, medial } \\
\text { maxillary sinus, ethmoid sinus, and/or sphenoid sinus }\end{array}$ \\
\hline Group II & SNIP involvement of lateral to medial maxillary wall \\
\hline Group III & SNIP involvement of the frontal sinus \\
\hline Group IV & SNIP involvement beyond the sinonasal cavities \\
\hline \multicolumn{2}{|c|}{ Kamel (21) } \\
\hline Type I & $\begin{array}{l}\text { SNIP's origin is from septum or lateral nasal wall (inferior } \\
\text { turbinate, middle turbinate, bulla ethmoidalis, uncinate } \\
\text { process, maxillary ostium, posterior ethmoid, sphenoid } \\
\text { sinus, frontal sinus) }\end{array}$ \\
\hline Type II & SNIP's origin is from maxillary sinus \\
\hline
\end{tabular}

\section{Surgery}

All patients underwent computer-assisted endoscopic resection of the SNIP by the senior author (ARJ). CT scans were evaluated preoperatively to identify the location of the tumour and confirm involved sinuses. Any tumour bulk extending into the nasal cavity was first removed, typically with a microdebrider blade (afterwards, specimen was sent for histopathology). A large number of cases involved the maxillary sinus exclusively, and in such cases, an uncinectomy and medial maxillectomy \pm anterior ethmoidectomy was performed. In the majority of cases, surgical resection involved debulking of the tumour in a piece-meal fashion within the involved sinuses, in conjunction with opening of all ipsilateral sinuses encasing the tumour bulk. The tumour was resected until the stalk of the tumour, if possible, was identified. This was confirmed, where possible, using intraoperative image guidance to correlate with area of hyperostosis on the CT images. Diseased mucosa and mucosa surrounding the tumour stalk was resected in order to obtain bony exposure. A diamond burr drill was then used to polish the bone at the site of the tumour stalk. Biopsies of the tissue surrounding the area 
Table 2. Recurrence rates based on staging system classifications (Krouse, Oikawa (modified Krouse), Cannady, Han, and Kamel), age group stratifications, and smoking history.

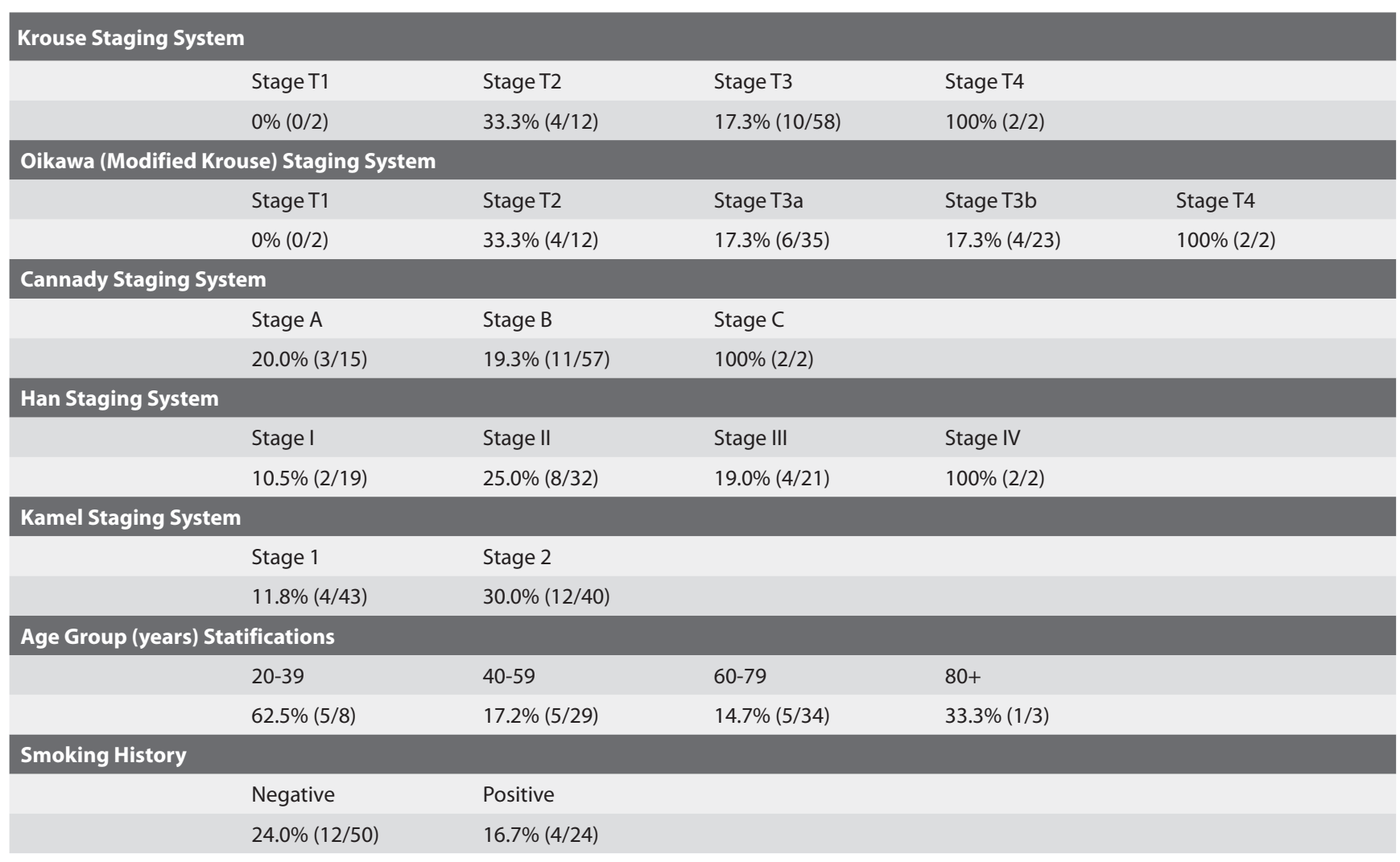

of suspected tumour attachment were sent as an intraoperative pathologic specimen in order to confirm negative margins after drilling. The site was irrigated and absorbable dressing (Surgiflo ${ }^{\oplus}$ ) was used to cover the site of bony exposure. No packing was placed. Tissue samples were then sent to the histopathology department for confirmation of the SNIP diagnosis. All patients were seen 1 week postoperatively, followed by standard clinical evaluation every 6 weeks for the first year, and then every 3 to 6 months for a minimum of 5 years where possible.

\section{Statistical analysis}

For statistical analyses, MedCalc Statistical Software version 17.7.2 (MedCalc Software bvba, Ostend, Belgium; http://www. medcalc.org; 2017) was used. The data was stratified between primary and secondary cases. Recurrence rates of SNIPs at St. Paul's Sinus Centre were calculated and compared between the two different types of cases. Mean times to recurrence were calculated and compared in a likewise manner. Additionally, stratification of patients based on age was completed to identify any association with recurrence risk. To evaluate the possible connection of smoking to recurrence, an odds ratio was calculated using any patient reported smoking history as a risk factor. To compare the different staging systems, a survival analysis was conducted using a Kaplan-Meier curve and a logrank test.
The survival analysis used the date of surgery as the start point and the recurrence date or last recurrence free follow-up as the endpoint. The same process was completed to compare the age-stratified patient groups.

\section{Results}

From 2008 to 2016, a total of 74 patients were treated for SNIPs at St. Paul's Sinus Centre, making up the study group. There were 52 primary cases - SNIPs that were managed surgically for the first time. 22 were secondary cases, defined as patients that had previously been managed surgically at another centre and demonstrated recurrence of the tumour. The mean age at the time of surgery was 58.7 years [age range 20 to 88]. Of the study population, 52 were male (70.3\%) and 22 were female (29.7\%). In terms of risk factors, a total of 24 patients had a smoking history, defined as either smoking at the time of surgery, or having smoked previously. Of those who had a smoking history, 15 were males and 9 were females. The remaining 50 patients had reported that they had never smoked. To monitor for recurrence, the mean time of follow-up was 42.3 months [Range: 3-105 months].

The majority of the tumours were localized to the maxillary sinus (54.1\%). The remaining tumours were localized to the sphenoid sinus (12.2\%), ethmoid sinus (14.9\%), frontal sinus 


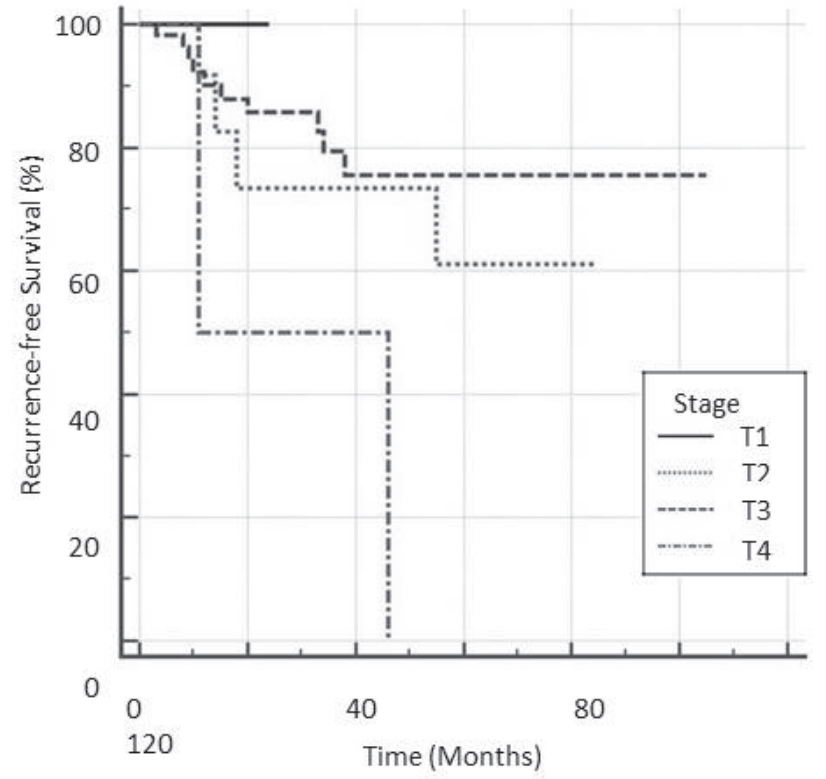

Figure 1. Kaplan Meier survival curve based on Krouse staging system.

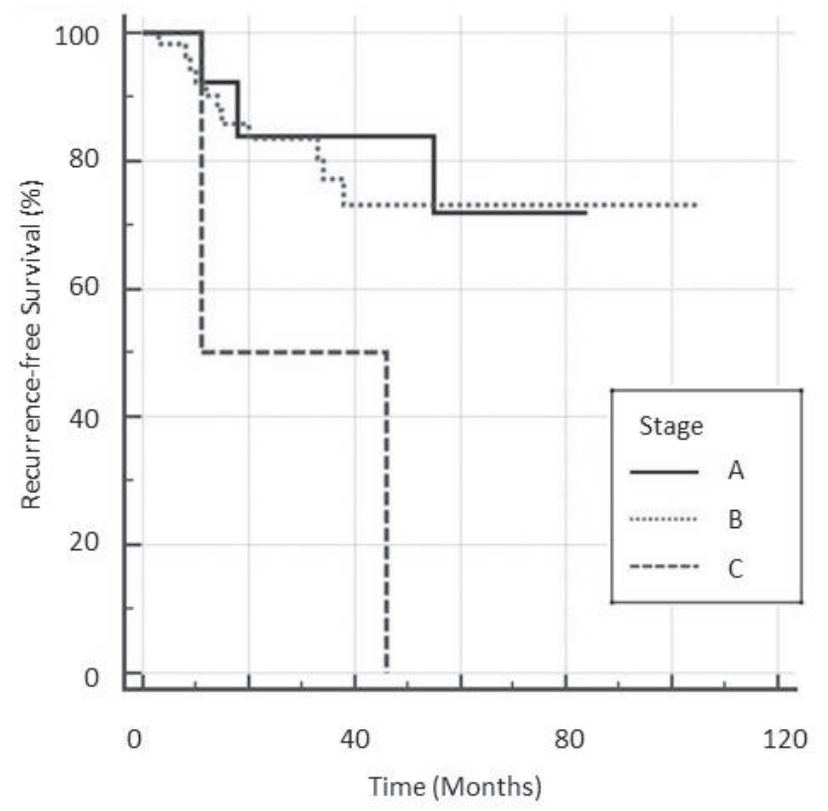

Figure 3. Kaplan Meier survival curve based on Cannady staging system.

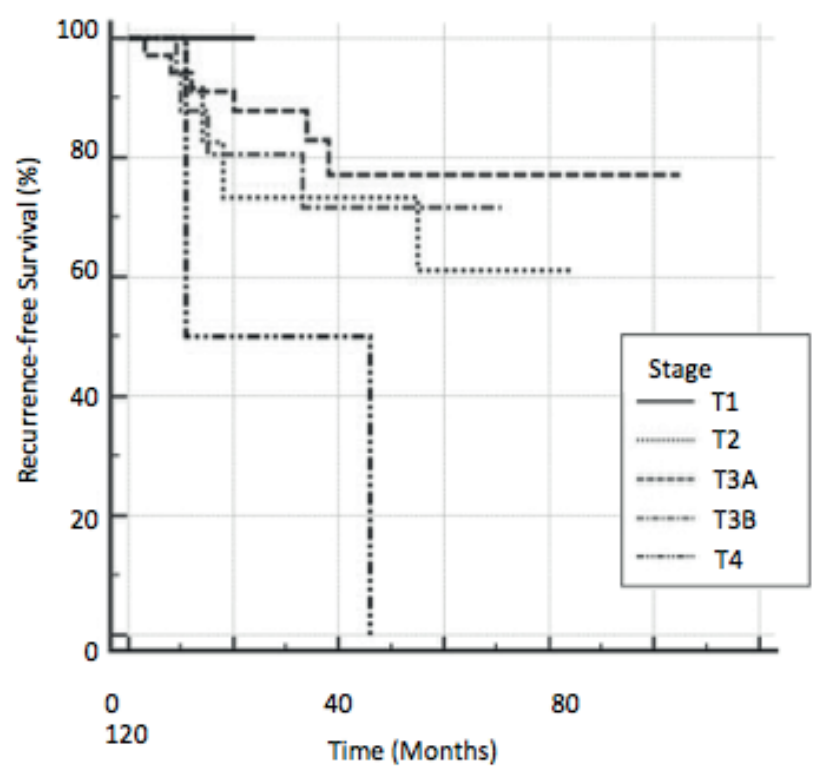

Figure 2. Kaplan Meier survival curve based on Oikawa staging system.

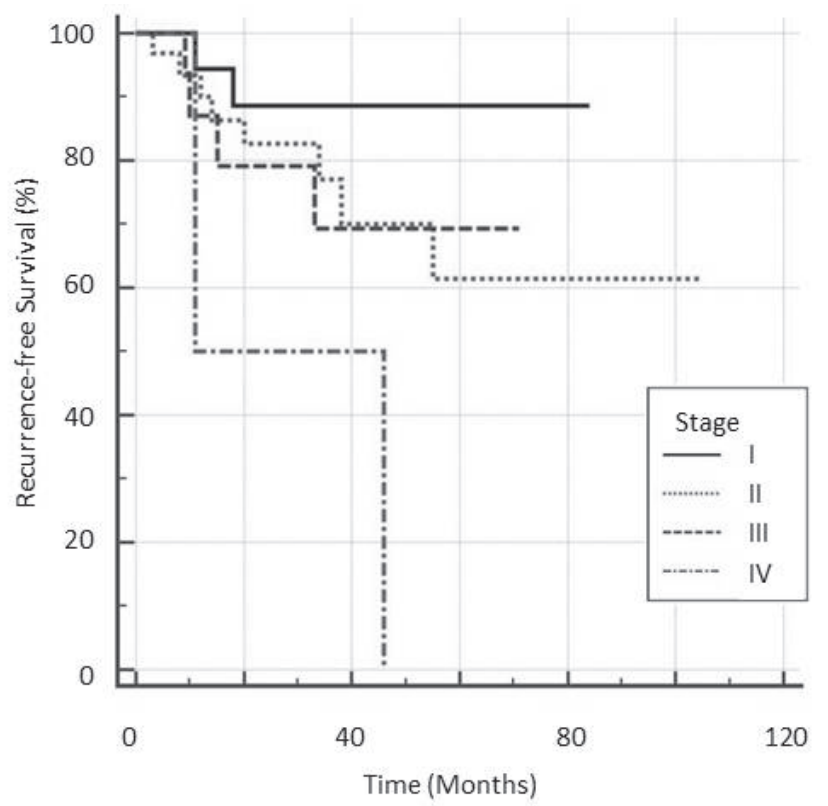

Figure 4. Kaplan Meier survival curve based on Han staging system.
(13.5\%), and septum (5.4\%). All cases underwent computer-assisted endoscopic resection, with 2 cases requiring the addition of an external approach (2.7\%).

A total of 16 patients had recurrences (21.6\%). 7 occurred within the first year. The following consecutive years featured 4, 2, 2 , and 1 recurrences respectively. The mean follow-up time until recurrence was 21.1 months [Range: 3-55 months]. 11 of them were primary cases and 5 were secondary. The recurrence rates for primary and secondary cases were $21.1 \%$ and $22.7 \%$ respectively. The mean follow-up time until recurrence for primary and secondary cases was 24.0 months [Range: 3-55 months] and
14.6 months [Range: 10-20 months] respectively. Recurrence rates according to origin site were maxillary (27.5\%), sphenoid (11.1\%), ethmoid (18.2\%), frontal (20.0\%), and septum (0.0\%). Recurrence rates for patients age 20 to 39,40 to 59,60 to 79 , and over 80 years old, were $62.5 \%, 17.2 \%, 14.7 \%$, and $33.3 \%$ respectively. Furthermore, of the 16 recurrences, 4 patients had a history of smoking. Smoking history had an odds ratio of 0.63 [Cl 95\%: 0.18-2.22].

Patients were distributed across Krouse, Oikawa (Modified Krouse), Cannady, Han, and Kamel staging systems, and recurrence rates were calculated for each stage of the respective systems. 


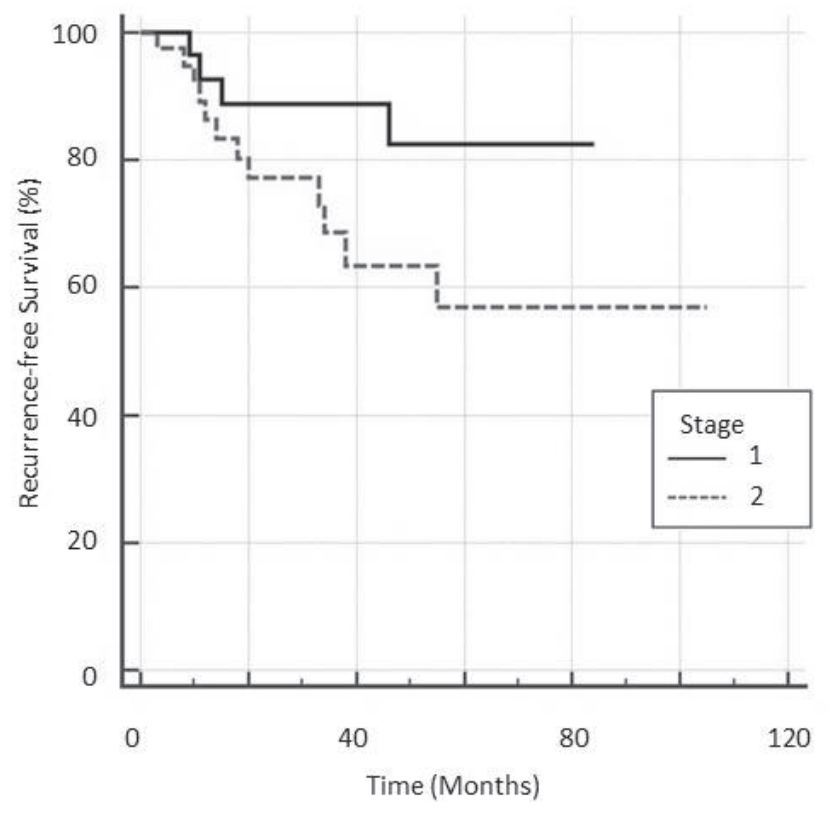

Figure 5. Kaplan Meier survival curve based on Kamel staging system.

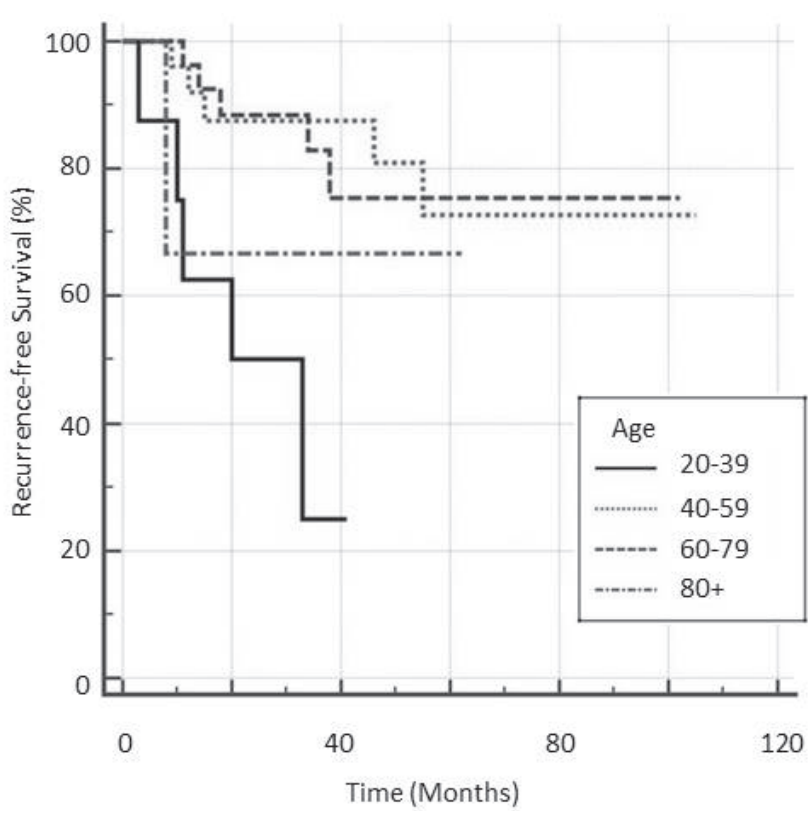

Figure 6. Kaplan Meier survival curve based on age stratification.
(Table 2). Generally, for each staging system, higher stages correlated with higher recurrence rates, with notable exceptions. Krouse T2 had a higher recurrence rate than T3. Similarly, Oikawa $\mathrm{T} 2$ had a higher recurrence rate than either T3A or T3B. Additionally, Han Stage II featured higher recurrence rates than Stage III. Recurrence rates based on age group stratification and smoking history were calculated (Table 2). The highest recurrence rates were found between ages 20 to 39.

Kaplan-Meier survival curves, which represent the recurrences over time for each stage, were generated for each of the staging systems (Figures 1-5). The logrank tests results from the analyses yielded the following chi-squared values: Krouse $6.73(p=0.081)$, Oikawa $7.02(p=0.13)$, Cannady $6.19(p=0.045)$, Han 8.23 $(p=0.042)$, and Kamel $3.29(p=0.070)$. An age-stratified KaplanMeier survival curve was graphed for visual representation (Figure 6). The chi-squared value from the age-stratified patient groups analysis was $12.79(p=0.0051)$.

\section{Discussion}

Many of the demographic results seen in this study support the current understanding of SNIPs. The gender demographic of our study - 52 males and 22 females - reinforces that males are at a higher risk for SNIPs ${ }^{(2)}$. Similarly, $54.1 \%$ of the SNIPs (40/74) originated from the maxillary sinus. This has been consistently demonstrated as the sinus that is most likely to be involved ${ }^{(1-3,22)}$. On the contrary, tumours that originate from the septum are usually a minority of cases ${ }^{(1-3,22)}$. In our study, there is a comparatively higher number (4/74 or $5.4 \%$ ) of septal origin.

The overall recurrence rate for SNIPs at our centre was $21.6 \%$. A recent meta-analysis on endoscopic recurrence rates by Goudakos et al. revealed SNIP recurrence to be 3 to $33 \%$, with a general recurrence of $13.8 \%{ }^{(23)}$. As such, this is comparable to other published studies ${ }^{(17,22,24,25)}$. However, this study's recurrence rate is relatively higher than recent studies, including Adriaensen et al. and Lombardi et al., who have reported recurrence rates less than $6 \%{ }^{(8,26)}$. Interestingly, there is limited variability in recurrence rates between primary and secondary cases $(21.1 \%$ vs. $22.7 \%)$. This was rationalized to the highly meticulous approach in endoscopic excision for each case, regardless of whether it was primary or secondary, with confirmation of complete removal verified by tumour negative margins. This was achieved by obtaining mucosal biopsies of the tissue surrounding the area of suspected tumour attachment. Biopsies were sent as an intraoperative specimen for frozen section histopathologic analysis in order to confirm negative margins after tumour removal and drilling.

Of the 16 patients that had recurrences, seven occurred within the first year. Other sites have also found that most recurrences occur within the first year post management ${ }^{(8,14)} .5$ of the 16 recurrences occurred beyond the 2 year post-surgery mark. The longest delay between surgery and recurrence was 55 months and occurred in a primary case. As such, this study provides further information about delayed recurrence of SNIPs, and supports recent recommendations for longer follow-up, including up to 6 years post-surgery ${ }^{(8,15,24)}$.

Of the age stratified groups, the 20 to 39 year old group had a higher recurrence rate of $62.5 \%$ (chi-squared $12.79, \mathrm{p}=0.0051$ ). However, there was not as large of a difference in recurrence rates between the other age groups. Other sites have similarly found an association with increasing age and decreasing risk of recurrence ${ }^{(27)}$.

There was no association between smoking history and recur- 
rence (OR 0.63, 95\% Cl:0.18-2.22). This is consistent with other published studies that have also been unable to confirm an association between SNIPs and smoking $(2,11,12)$. In terms of staging systems for this condition, Krouse et al. proposed a staging system in 2000 with the aim to uniformly measure the extent of disease, which also acts as a tool to be used in outcomes research. However, it was not developed to evaluate recurrence ${ }^{(16)}$. Using the Krouse staging system criteria for the cases in our study, the majority (78.4\%) were Stage T3 (58/74). Stage T3 is defined based on involvement of the frontal, sphenoid, or maxillary sinus beyond the medial wall ${ }^{(16)}$. The chi-squared value of $6.73(p=0.081)$, was insignificant; however, the recurrence rates for $\mathrm{T} 1(0 \%)$ and $\mathrm{T} 4(100 \%)$ were distinct from other stages. With further inspection, recurrence rates for T2 (33.3\%) and T3 (17.3\%) were not clearly distinguished and did not correlate with the stage. In fact, there was a higher rate of recurrence in Stage T2 than Stage T3 patients. Based on these results, it appears that this staging system does not provide good distinction between intermediately staged SNIPs for prognostic purposes. This is contrary to the meta-analysis results by Lisan et al., which found a significantly higher recurrence risk for T3 when compared with $\mathrm{T} 2{ }^{(18)}$.

Oikawa suggested further subdivision of the Krouse staging system based on magnetic resonance imaging (MRI) studies. The goal was to identify patients who require different surgical techniques due to their risk of recurrence post surgery ${ }^{(20)}$. The staging system deviates from the one by Krouse through the distinguishment of T3B from T3A using a criterion of frontal sinus or supraorbital recess involvement ${ }^{(20)}$. This is useful as the majority of cases are T3 in the Krouse staging system. The Oikawa system provides a way to separate different cases within this stage ${ }^{(20)}$. Oikawa et al. proposed that $\mathrm{T} 3$ should be subdivided into two groups preoperatively, as T3B tumours featured locations difficult to access, leading to potentially higher rates of recurrence ${ }^{(20)}$. However, the logrank test of this study resulted in a non-significant chi-squared value of $7.02(p=0.13)$. Accordingly, this staging system does not appear to have good prognostic value. Similar to Krouse, the Oikawa system does not sufficiently distinguish between $\mathrm{T} 2, \mathrm{~T} 3 \mathrm{~A}$, and T3B patients for recurrence.

Cannady created a staging system that is based on recurrences, to predict recurrence of SNIPs after advanced endoscopic techniques ${ }^{(19)}$. The system uses involvement of the sphenoid, frontal, and beyond the medial maxillary wall to identify Group B cases from Group $A^{(19)}$. The system then uses extension outside the sinus and parasinuses to define Group $C$ cases ${ }^{(19)}$. Using this criterion, the cases in our study report a statistically significant chi-squared value: 6.19 ( $p=0.045)$. This was a result of the differentiation of the Group C recurrence rate (100\%). Groups A and $B$, which were defined based on different parasinus involvement had recurrence rates for Group A (20.0\%) and B (19.3\%). Conse- quently, these groups were not clearly differentiated from each other when it came to recurrence.

Han proposed a different staging system based on "trends in diagnosis and treatment" ${ }^{\prime 1}$. Analysis of our cases indicated a chi-squared value of 8.23 ( $p=0.042$ ). This large and statistically significant chi-squared value reflects the Han staging system's distinction between the recurrence rate for Stage I (10.5\%) and Stage IV (100\%). However, the staging system did not provide the same separation for Stages II and III, with recurrence rates of $25.0 \%$ and $19.0 \%$, respectively. Looking in depth, a Han Stage III SNIP has frontal parasinus involvement, while a Stage II does not. It is evident that this stage classification is based on tumour involvement of different parasinuses and derives from the difficulty associated with surgically accessing these parasinuses, particularly the frontal sinus ${ }^{(1)}$. Acknowledging this idea and based on the results of this study, classification based on parasinus involvement did not correlate well with different recurrence rates.

Kamel created a relatively simple staging system to evaluate for a suitable surgical technique for patients. It distinguishes between SNIPs that originate from the maxillary sinus (Type 2) from those that do not (Type 1) ${ }^{(21)}$. The recurrence rates for Type $1(11.8 \%)$ and Type 2 (30\%) correlated well to illustrate the difference in outcomes between the stages. Given the simplicity of the staging system and its ability to correlate well with recurren$\mathrm{ce}$, the Kamel staging system may have the greatest versatility. Nonetheless, while the trend is apparent, the staging system did not produce a statistically significant chi-squared value of $3.29(p=0.070)$. As such, it was similar to other systems, in which differentiation of SNIPs by specific parasinus origin did not yield statistically significant prediction of recurrences.

Overall, based on the survival analysis and logrank tests alone, the Cannady and Han staging systems demonstrate ability to predict recurrence risks most accurately. Equally, within all the investigated staging systems, whenever a system relies upon a specific parasinus localization of the SNIP to differentiate between two comparable stages, the recurrence rates were very similar, and there was poor differentiation between groups. This was epitomized with Krouse (T2 vs T3), Oikawa (T2 vs T3A/B), Cannady (A vs B), and Han (II vs III). These staging scales were largely influenced by previous surgical technique and experience, in which certain locations were more difficult to access, and therefore harder to carry out a successful resection. However, the endoscopic approach has become the gold standard for resecting SNIPs over the last decade. Significant advancements in surgical techniques, increase in experience, and perfection of endoscopic skills have continued to drive down recurrence rates. The difficulty of accessing these specific sinuses may have become a less significant factor over time. As such, staging systems that rely on specific parasinus tumour origins to differentiate between comparable tumour stages may be less effective in 
predicting recurrence in this present era.

It should be acknowledged that there were limitations to this study. As a retrospective chart review, many factors could not be controlled. Despite attempts for a minimum 5-year follow-up, patient factors resulted in nonideal durations. Staging was completed by a single blinded rhinologist. Preferably, it would be two independent reviewers coming to an agreement. Detailed origin sites of the original tumour and recurrence were also not recorded. This limited identification of sinus areas prone to recurrence. Another limitation was the application of Oikawa staging system. The intent of the Oikawa system was to showcase the benefits of pre-operative staging ${ }^{(20)}$. The original study used $M \mathrm{MI}^{(20)}$. However, only CT imaging was available in this retrospective study. Thus, results should be considered accordingly. The conclusions drawn for each of the age stratifications and staging systems had a potential for misrepresentation. This was attributed to unequal patient distribution across age groups and stages for each system. Specifically, the extremes within each staging system had limited patient numbers, and interpretation of these results should take this into consideration.

\section{Conclusion}

The recurrence rate of $21.6 \%$, found in this study, is comparable to published literature. Additionally, there was no significant differences in the recurrence rates between primary and secondary cases. The age group of 20-39 years featured the highest rates of recurrence when investigating the impact of age on recurrence. No statistically significant association was found between smoking and recurrence. Han and Cannady staging systems were found to define patient groups that correlated well with recurrence. However, staging systems that differentiate comparable stages based on involvement of specific parasinuses were not found to have good prognostic abilities for recurrence. Nonetheless, staging systems should continue to play a role in the management of SNIPs, especially to identify patients requiring additional post-surgical monitoring.

\section{Acknowledgement}

Thank you to the St. Paul's Sinus Centre research team for their support.

\section{Authorship contribution}

WM and DW collected, analyzed, and interpreted the data. SA was the rhinologist who staged all cases. $S A, A D$, and ARJ mentored and supervised data interpretation. All authors contributed to the writing and revision of the manuscript.

\section{Conflict of interest}

The authors declared that no conflict of interest exists.

\section{References}

1. Han JK, Smith TL, Loehrl T, Toohill RJ, Smith MM. An Evolution in the Management of Sinonasal Inverting Papilloma. Laryngoscope 2001; 111: 1395-1400.

2. Lisan Q, Laccourreye O, Bonfils P. Sinonasal inverted papilloma: From diagnosis to treatment. Eur Ann Otorhinolaryngol Head Neck Dis 2016; 133: 337-341.

3. Lawson W, Patel ZM. The evolution of management for inverted papilloma: an analysis of 200 cases. Otolaryngol Head Neck Surg 2009; 140: 330-335.

4. Klimek T, Atai E, Schubert M, Glanz H. Inverted papilloma of the nasal cavity and paranasal sinuses: clinical data, surgical strategy and recurrence rates. Acta Otolaryngol 2000; 120: 267-272.

5. Diamantopoulos II, Jones NS, Lowe J. All nasal polyps need histological examination: an audit-based appraisal of clinical practice. J Laryngol Otol 2000; 114: 755-759.

6. Khandekar S, Dive A, Mishra R, Upadhyaya N. Sinonasal inverted papilloma: A case report and mini review of histopathological features. J Oral Maxillofac Pathol 2015; 19: 405

7. Wang M-J, Noel JE. Etiology of sinonasal inverted papilloma: A narrative review. World Journal of Otorhinolaryngology Head and Neck Surgery 2017; 3: 54-58.

8. Adriaensen GFJPM, Lim K-H, Georgalas C,
Reinartz SM, Fokkens WJ. Challenges in the Management of Inverted Papilloma: A Review of 72 Revision Cases. Laryngoscope 2016; 126: 322-328.

9. Zhao R-W, Guo Z-Q, Zhang R-X. Human papillomavirus infection and the malignant transformation of sinonasal inverted papilloma: A meta-analysis. J Clin Virol 2016; 79 : 36-43.

10. Lawson W, Schlecht NF, Brandwein-Gensler $M$. The role of the human papillomavirus in the pathogenesis of Schneiderian inverted papillomas: an analytic overview of the evidence. Head Neck Pathol 2008; 2: 49-59.

11. Thompson L. World Health Organization classification of tumours: Pathology and genetics of head and neck tumours. New York: WHO Publications Center, 2006; 85: 74.

12. Buchwald C, Franzmann MB, Tos $M$. Sinonasal papillomas: a report of 82 cases in Copenhagen County, including a longitudinal epidemiological and clinical study. Laryngoscope 1995; 105: 72-79.

13. Reh DD, Lane AP. The role of endoscopic sinus surgery in the management of sinonasal inverted papilloma. Curr Opin Otolaryngol Head Neck Surg 2009; 17: 6-10.

14. Philpott CM, Dharamsi A, Witheford M, Javer AR. Endoscopic management of inverted papillomas: long-term results--the St. Paul's Sinus Centre experience. Rhinology 2010; 48: 358-363.
15. Kim D-Y, Hong $S-L$, Lee $C H$, Jin $H-R$, Kang JM, Lee B-J, et al. Inverted papilloma of the nasal cavity and paranasal sinuses: a Korean multicenter study. Laryngoscope 2012; 122: 487-494.

16. Krouse JH. Development of a staging system for inverted papilloma. Laryngoscope. 2000; 110: 965-968.

17. Gras-Cabrerizo JR, Montserrat-Gili JR, Massegur-Solench H, León-Vintró X, De Juan J, Fabra-Llopis JM. Management of sinonasal inverted papillomas and comparison of classification staging systems. Am J Rhinol Allergy 2010; 24: 66-69.

18. Lisan Q, Moya-Plana A, Bonfils P. Association of Krouse Classification for Sinonasal Inverted Papilloma With Recurrence: A Systematic Review and Meta-analysis. JAMA Otolaryngol Head Neck Surg 2017; 143: 1104-10.

19. Cannady SB, Batra PS, Sautter NB, Roh H-J, Citardi MJ. New staging system for sinonasal inverted papilloma in the endoscopic era. Laryngoscope 2007; 117: 1283-1287.

20. Oikawa K, Furuta Y, Nakamaru Y, Oridate N, Fukuda S. Preoperative staging and surgical approaches for sinonasal inverted papilloma. Ann Otol Rhinol Laryngol 2007; 116: 674-680.

21. Kamel RH. Transnasal endoscopic medial maxillectomy in inverted papilloma. Laryngoscope 1995; 105: 847-853. 
22. Sciarretta V, Fernandez IJ, Farneti P, Pasquin E. Endoscopic and combined externaltransnasal endoscopic approach for the treatment of inverted papilloma: analysis of 110 cases. Eur Arch Otorhinolaryngol 2014; 271: 1953-1959.

23. Goudakos JK, Blioskas S, Nikolaou A, Vlachtsis K, Karkos P, Markou KD. Endoscopic Resection of Sinonasal Inverted Papillomas: Systematic Review and MetaAnalysis. Am J Rhinol Allergy 2018; 01: 194.

24. Chai CK, Tang IP, Prepageran N. A Review of Inverted Papilloma at a Tertiary Centre: A Six-Year Experience. Int J Otolaryngol and
Head Neck Surg 2013; 02: 156

25. Busquets JM, Hwang PH. Endoscopic resection of sinonasal inverted papilloma: a meta-analysis. Otolaryngol Head Neck Surg 2006; 134: 476-482.

26. Lombardi D, Tomenzoli D, Buttà L, Bizzon A, Farina D, Sberze F, et al. Limitations and complications of endoscopic surgery for treatment for sinonasal inverted papilloma: a reassessment after 212 cases. Head Neck Surg 2011; 33: 1154-61.

27. Healy DY, Chhabra N, Metson R, Holbrook EH, Gray ST. Surgical risk factors for recurrence of inverted papilloma. Laryngoscope
2016; 126: 796-801.

Dr. Amin R. Javer

Rm 2600-1081 Burrard Street

Vancouver, BC

V6Z 1Y6, Canada

Tel: +1 (604) 806-9926

Fax: +1 (604) 906-9690

E-mail:sinusdoc@me.com 